\title{
Short time delay does not hinder haptic communication benefits
}

\author{
Ekaterina Ivanova ${ }^{1}$ Jonathan Eden $^{1}$, Silei Zhu ${ }^{2}$, Gerolamo Carboni ${ }^{1}$, Aaron Yurkewich $^{1}$, Etienne Burdet ${ }^{1}$
}

\begin{abstract}
Haptic communication, the exchange of force and tactile information during dancing or moving a table together, has been shown to benefit the performance of human partners. Similarly, it could also be used to improve the performance of robots working in contact with a human operator. As we move to more robot integrated work spaces, how common network features such as delay or jitter impact haptic communication is unknown. Here using a human-like interactive robotic controller, that has been found to be indistinguishable by humans to human interaction, we evaluate how subjects' performance and perception is altered by varying levels of transmission delay. We find that subjects are able to recognise haptic delay at very small levels within haptic interaction. However, while they are consciously aware of the delay they can only compensate for it up until a certain point, after which they perceive it as the addition of noise/impedance into the system.
\end{abstract}

\section{INTRODUCTION}

From everyday actions such as dancing, to more specialised activities like moving a sofa, we often use force transmission and/or haptic interaction with one another to complete tasks. This force transmission can be used when a single person cannot achieve the task alone, when safety needs to be ensured or to communicate information between people. Specifically, the transmission of force for the communication of information (haptic communication) has recently been shown to improve performance irrespective of relative partner skill [1], [2].

As robots become increasingly demanded within the work place, their integration with human labour will require that they can exploit similar benefits as observed from humans working together. How to best coordinate robots working with humans has been well studied within haptic shared control [3]. However, such mechanisms until recently did not account for the human-like features of haptic communication. Recently, an algorithm has been developed to mimic the features of human haptic communication thereby enabling more human-like performance in haptic human-robot interaction [4]. Unlike traditional robotic guidance, this algorithm preserves the benefits resulting from haptic communication for both partners regardless of the partner skill. Furthermore, it has been shown that the resulting interaction with the robot is not felt as different to haptic interaction with a human [5].

*This work was supported in part by the EU H2020 grants ICT871803 CONBOTS, MSCA-843408 TRIMANUAL, FETOPEN-829186 PH-CODING and ITN-861166 INTUITIVE, as well as by the UK EPSRC EP/R026092/1 FAIRSPACE.

**Corresponding authors: \{e.ivanova17, e.burdet\}@imperial.ac.uk

${ }^{1}$ Ekaterina Ivanova, Jonathan Eden, Gerolamo Carboni, Aaron Yurkewich and Etienne Burdet are with Imperial College of Science, Technology and Medicine, London, UK China
The introduction of robots working in conjunction with humans also leads to the potential for timing issues caused by transmission over a network or with limited computational processing. For robots, the impact of delay is well studied where it is known that delay leads to decreased performance and the potential for instability [6]. However, appropriate model knowledge predictions made using tools such as Kalman filters [7] or Smith predictors [8] are able to mitigate some of the impact of delay.

For human subjects, the impact of delay has also been investigated, where its effect on haptic communication has not been considered. For visual delay, it has been shown that the impact is task and trajectory dependent [9], [10]. In reaching tasks, Mackenzie and Ware first showed that subjects can compensate for delay and still perform with relatively small error. This is because subjects increase moving time to account for the effect of delay, where the change in movement time could be modelled through a delay dependent extension of Fitts's law [11]. For small visual delays, it was found that performance is quite variable, however, subjects can have reduced movement time relative to delay free conditions [9] potentially due to robustness in the allotted deceleration time for the movement. Increasing the delay then results in performance that continues to degrade until for values greater than $100 \mathrm{~ms}$. The effect stabilises once the subject is able to consciously recognise a delay [12]. In tracking tasks, the strategy of increased movement time is not possible and therefore subjects have to actively counteract delay. Delay cancellation has been shown to be possible until a certain trajectory and task dependent delay threshold at which there is a steep drop-off in performance due to the subject's inability to account for the delay [10].

To our knowledge, the effect of haptic delay has not been well studied for tracking tasks relying on continuous feedback control. It has however been considered for reaching tasks both in combination to and in isolation of delays in visual feedback. In each case, the results suggest similar effects to that of visual delay. However, the results with only haptic delay suggest that humans may be more sensitive, such that their performance degrades at smaller levels of latency [12], [13].

In this work we look to understand the impact of delayed force exchange on haptic communication. We study this by observing the interaction between a human subject and a human-like robotic partner of [4], in which varying levels of onset delay are imparted. We first ask when are subjects engaging in haptic communication able to recognise the presence of delay. With this knowledge we then consider if the subjects are able to compensate for the effects of delay. 
And whether their capability to compensate is affected by their level of conscious awareness of the delay.

\section{METHODS}

The experiment was approved by the Research Ethics Committee of Imperial College London and carried out by 20 subjects ( 10 female) without known sensorimotor impairment aged $24.42 \pm 3.58$ years old. One participant was left-handed. Each subject was informed about the experiment, gave informed consent, and filled in the Edinburgh handedness form [14] as well as a demographic questionnaire before starting with the experiment. Subjects carried out the experiment in pairs, so that they did not know whether they were interacting with a robot or human. Each of them interacted with an individual handle of the Hi5 dual robot [15], which is a 1-DOF flexion/extension wrist interface that can be controlled by both a human subject or robotic controller (Fig.1A). They were separated by a curtain to prevent visual communication. The Hi5 interface was controlled at $1000 \mathrm{~Hz}$, while wrist angle data was recorded at $100 \mathrm{~Hz}$.

Each subject was asked to track a moving target "as accurately as possible" using the wrist flexion/extension of their dominant hand (Fig.1A). The target trajectory (in degrees) was given by

$$
\begin{aligned}
q^{*}(t) & \equiv 18.5 \sin \left(\frac{\pi\left(t+t_{0}\right)}{1.547}\right) \sin \left(\frac{\pi\left(t+t_{0}\right)}{2.875}\right) \\
0 & \leq t \leq 30 s
\end{aligned}
$$

where to prevent a strong pattern across trials, each trial started from a randomly selected starting time $\left\{t_{0} \in\right.$ $\left.[0,30] \mathrm{s} \mid q^{*}\left(t_{0}\right) \equiv 0\right\}$.

A
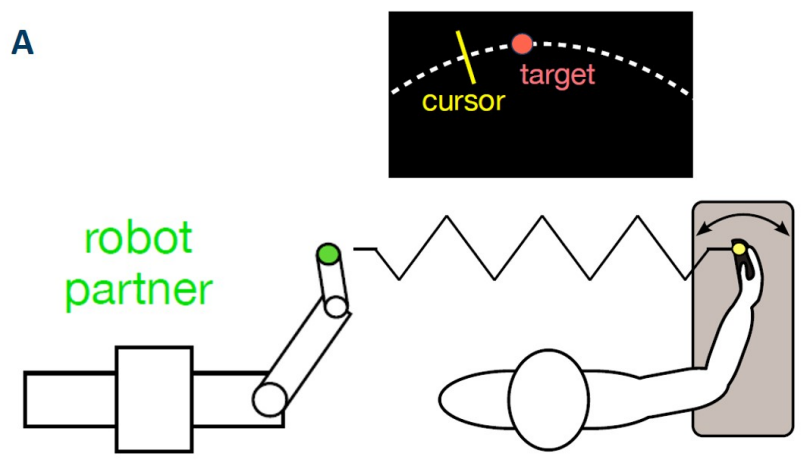

B

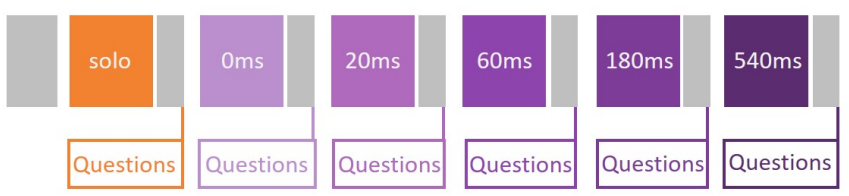

Fig. 1. Experiment description. A: Subjects track a randomly moving target with their wrist flexion/extension movement while either being independent or connected to a reactive robot partner (RP) through a robotic interface Hi5. The experimental protocol is shown in B. The grey boxes represent the familiarisation/washout trials and the colourful blocks are the experimental conditions. The order of the blocks was the same for all subjects.
The tracking task was performed in either a free or robot assistance condition using interaction with a human-like reactive controller [4], [5] that mimics human-like interaction behaviour, including differences in skill. This is achieved through a sensory augmentation approach in which the information coming from the haptic connection is used to infer the partner's target [4], which is then combined with own target in a stochastically optimal manner. Within the assistance condition, the Hi5 interface provided an elastic torque (in $\mathrm{Nm}$ ) on the wrist

$$
\begin{aligned}
\tau(t) & =1.72\left[q_{r}\left(t_{\delta}\right)-q\left(t_{\delta}\right)\right]+0.0286\left[\dot{q}_{r}\left(t_{\delta}\right)-\dot{q}\left(t_{\delta}\right)\right] \\
t_{\delta} & \equiv t-\delta
\end{aligned}
$$

where the time was delayed by $\delta \geq 0$, reflecting a delay in the exchange of forces, and $q_{r}$ denotes the robotic partner (RP) angle computed by the human-like controller. The damping and stiffness constants were chosen to match the conditions of medium stiffness in [4], since at these parameters an interaction with a RP is clearly perceived by participants and the connection is compliant [5].

Surface electrodes were used to record electromyographical (EMG) activity from the flexor carpi radialis (FCR) and extensor carpi radialis longus (ECRL) muscles. This was calibrated through a process in which subjects were asked to flex/extend their wrist while their wrist was locked by the device at $0^{\circ}$ corresponding to the subject's most comfortable position. The subject was asked to produce flexion and extension torques of 1, 2, 3 and $4 \mathrm{Nm}$ for 2 seconds, first flexion then extension, with a rest period of 5 seconds between each activation to prevent fatigue. This EMG data was linearly regressed with torque to estimate the relationship between muscular activity and torque. Then co-contraction was computed as

$$
u(t) \equiv \min \left\{\tau_{f}(t), \tau_{e}(t)\right\},
$$

where $\tau_{f}(t)$ and $\tau_{e}(t)$ are the (absolute) flexor and extensor torque, computed from the respective EMG signals. The average co-contraction over all participants was computed from each participant's normalised co-contraction, calculated as

$$
u_{n} \equiv \frac{\bar{u}-\bar{u}_{\min }}{\bar{u}_{\max }-\bar{u}_{\min }}, \quad \bar{u} \equiv \frac{1}{T} \int_{0}^{T} u(t) d t, \quad T=30 s
$$

with $\bar{u}_{\min }$ and $\bar{u}_{\max }$ the minimum and maximum of the means of all trials of the specific participant.

The experiment protocol is described in Fig.1B. In the initial solo block each subject attempted 5 trials of the task without a haptic connection to be familiarised with the task and to minimise subsequent learning effects. In the main experiment, subjects carried out 6 blocks, each of 10 trials. Each block included 7 experimental trials followed by 3 washout trials of the solo condition. The first experimental condition was without any interaction and in the following 5 blocks assistance from a robot partner was introduced. The RP's motor noise deviation [4] was set after the solo block as equal to the deviation observed in the subject's tracking movement during the final solo trial, to ensure 
that the subject and the RP have similar skill level [16]. This was chosen as it has been found to provide the best performance [2]. Each trial took $30 \mathrm{~s}$ and was followed by a $5 \mathrm{~s}$ break. The delay within the robotic assistance trials was increased from each block to the next with values $\{0,20,60,180,540\} \mathrm{ms}$ and the sequence of the blocks was identical for each participant. These delay levels were chosen in order to include small delays values considered in [9] and values greater than the threshold for performance loss found in [10]. After each block, the subjects had to answer the questions in the caption of Fig. 2 on how they perceived the interaction.

To investigate delayed force exchange's effect on subject performance, Root-Mean-Square Error (RMSE), the smoothness metrics SPARC [17], cross-correlation delay and cocontraction were considered. The cross-correlation corresponds to the time interval between the target's movement and the subject's resulting motion and was calculated as the time lag at which the cross-correlation between the target and subject's positions was the highest. To understand how subjects perceived the changes in delay, a questionnaire composed of a 5-point Likert-scale items was analysed (see the question list in Supplementary Materials). For the analysis of all data, a non-parametric Friedman test of differences among repeated measures was conducted, since all metrics were not normally distributed. For the pairwise comparisons between single delay levels, a post-hoc paired Wilcoxon sign-rank test was employed and the Hommel adjustment was used to control the family-wise error rate. For the statistical analysis of objective values (RMSE, SPARC, cross-correlation delay, co-contraction) for each subject were averaged over all trials in a block.

\section{RESULTS}

\section{A. Questionnaire}

Could the subjects identify that there was haptic interaction? And how would they describe the interaction? The presence of forces was clearly identified by the subjects in all the interaction blocks. A Friedman test showed significant differences among the groups $\left(\chi^{2}(5)=59.644, p<\right.$ $0.0001)$, where post-hoc Wilcoxon tests revealed that in particular the solo block was distinguished from all other groups ( $p<0.004$ for all pairwise comparisons).

With respect to describing the interaction, connection to the partner was identified as providing more assistance than the solo execution of the task even with a relatively large delay, see Figure 2B $\left(\chi^{2}(5)=58.622, p<0.0001\right.$ for Friedman test, $p<0.02$ for pairwise comparisons of all interaction blocks with solo condition). However as the delay was increased, subjects saw the interaction as providing less assistance than when interacting without delay $(p<0.03$ for all comparisons of $0 \mathrm{~ms}$ with $60-540 \mathrm{~ms}$ delay).

Surprisingly, from Figure 2C subjects appear able to clearly identify the delay even for small delay values compared to the no-delay condition ( $p<0.05$ for pairwise comparisons between $0 \mathrm{~ms}$ and $20-540 \mathrm{~ms}$ delay groups). While the subjects identified delay they also associated the interaction with perturbations (Goodrman-Kruskal correlation $\gamma(118)=0.58, p<0.0001)$, resistance $(\gamma(119)=$ $0.60, p<0.0001)$ and noise $(\gamma(119)=0.43, p<0.0001)$.

Resistance was distinctly identified and increased at higher delay values (see Figure 2A): its perception was higher with any delay than no interaction $(p<0.05$ for all pairwise comparisons), with $60-540 \mathrm{~ms}$ delay than with delay free interaction ( $p<0.04$ for all pairwise tests) and with $540 \mathrm{~ms}$ delay the resistance was higher compared to all other delay levels $(p<0.03$ for all post-hoc tests between $540 \mathrm{~ms}$ and 20-180 ms delay groups). Although, the trend for feeling noise was very similar (Figure 2D) - growing with larger delay - only the 180-540 ms delay levels were significantly different from the solo condition (both $p<0.02$ ) and no-delay condition (both $p<0.03$ ). This can possibly be associated with a loss of predictability of the robot behaviour $(\gamma(119)=-0.48, p<0.0001$ for GoodmanKruskal correlation between delay and predictability scales).

\section{B. Performance}

How did the subjects perform with different interaction delays? Figure $3 \mathrm{~A}$ depicts the tracking error of the subjects for each experimental condition and Figure $3 \mathrm{~B}$ shows the median smoothness for each block.

A Friedman test revealed a significant effect of delay on tracking accuracy $\left(\chi^{2}(5)=63.143, p<0.0001\right)$. From the subject performance (see Figure 3A), it can be observed that, as expected, tracking performance is improved by interaction with the robotic partner $(Z=3.9199, p<0.0001$ for comparison between solo and $0 \mathrm{~ms}, Z=3.8079, p<0.0001$ between solo and $20 \mathrm{~ms}, Z=3.6959, p=0.0002$ between solo and $60 \mathrm{~ms}$ ). There is subsequently no significant difference found in the performance between delays of $0-60 \mathrm{~ms}$ (both $p>0.3$ ). After $60 \mathrm{~ms}$, however, the accuracy degrades resulting in worse performance with the $180 \mathrm{~ms}$ delay relative to the $0-60 \mathrm{~ms}$ range $(Z=-3.0613, p=0.0070$ between $0 \mathrm{~ms}$ and $180 \mathrm{mss}, Z=-3.7333, p=0.0002$ between $20 \mathrm{~ms}$ and $180 \mathrm{~ms}$ and $Z=-3.3973, p=0.001$ between $60 \mathrm{~ms}$ and $180 \mathrm{~ms}$ delay blocks). The performance continues to degrade at $540 \mathrm{~ms}$ which is significantly worse than the 0$180 \mathrm{~ms}$ delays ( $p<0.001$ for all pairwise comparisons) and even worse than the solo performance $(Z=-2.7626, p=$ 0.021 ). However, for the 0 to $180 \mathrm{~ms}$ delay blocks, accuracy was not worse than the performance without any interaction ( $Z=1.008, p=0.4090$ between $180 \mathrm{~ms}$ and no interaction).

Similar results were seen for movement smoothness, in Figure 3B $\left(\chi^{2}(5)=75.771, p<0.0001\right)$ : interaction with the RP improves the movement quality $(p<0.0001$ for comparisons between solo and $0,20 \mathrm{~ms}$, as well as $p=$ 0.0253 between solo and $60 \mathrm{~ms}$ delay blocks), but with an increasing delay the motion becomes less smooth $(p<$ 0.0001 for all pairwise comparisons between $180-540 \mathrm{~ms}$ with $0-20 \mathrm{~ms}$ delay blocks). In contrast to accuracy, from $60 \mathrm{~ms}$ delay onward the smoothness is significantly affected ( $Z=3.8453, p<0.0001$, for between 0 and $60 \mathrm{~ms}, Z=$ $3.8079, p<0.0001$ between 20 and $60 \mathrm{~ms}$ delay blocks). 

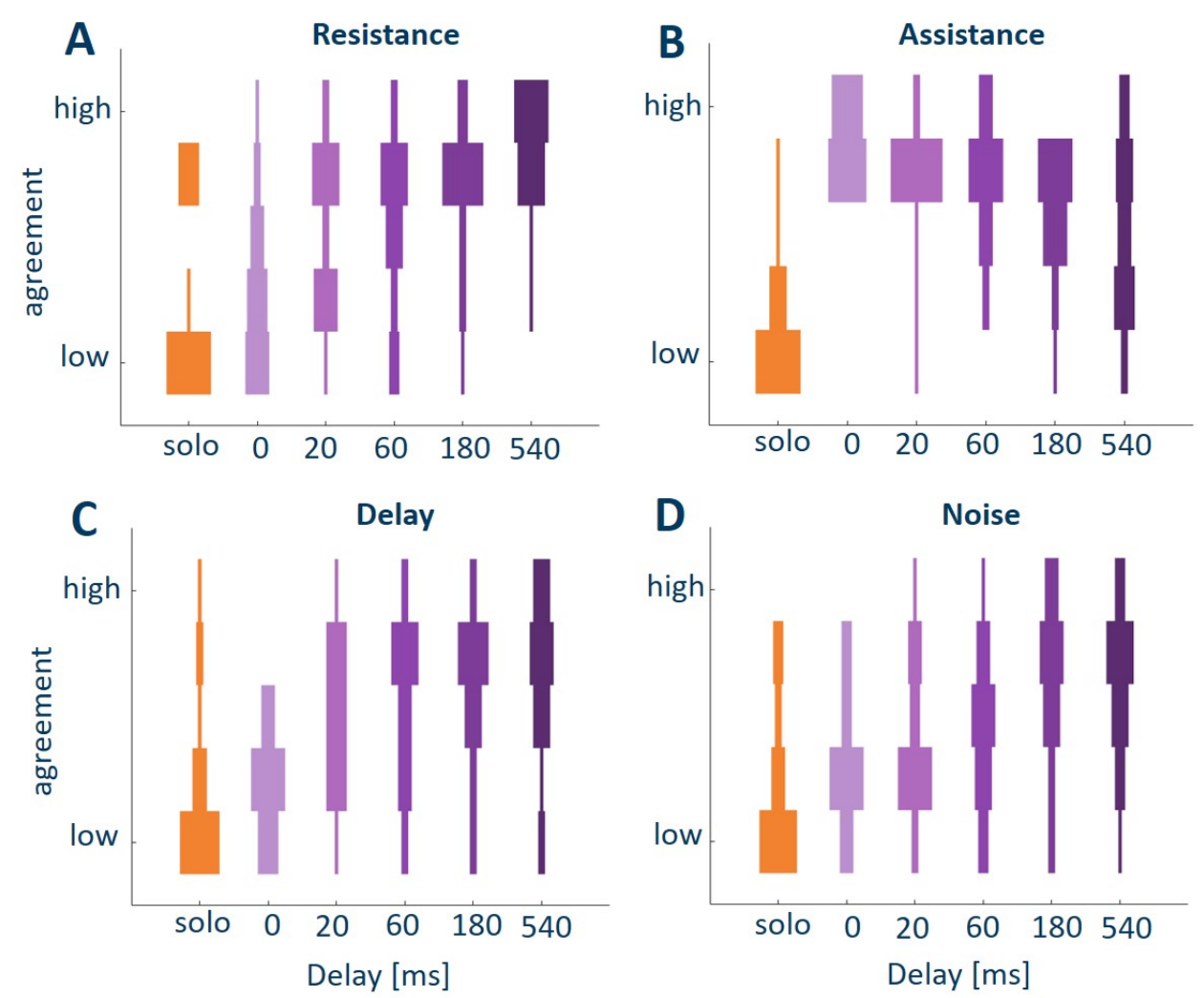

Fig. 2. Assessment of perception. Histograms of interaction assessment, where questions A-D were answered after each trial and $1 / 5$ corresponds to strongly disagree/agree. A: Resistance question "It seemed like I felt haptic resistance". B: Assistance question "It seemed like I felt haptic assistance". C: Delay question "It seemed like I felt haptic interaction with a delay". D: Noise question "It seemed like I felt haptic noise".

Interestingly, the effects on smoothness reverse in the washout trials: after interacting with a RP in the $180 \mathrm{~ms}$ and $540 \mathrm{~ms}$ conditions, subjects showed significantly better smoothness compared to the solo $(Z=-3.3599, p=0.004$ for comparison with $180 \mathrm{~ms}, Z=-3.2853, p=0.006$ with $540 \mathrm{~ms})$ and $20 \mathrm{~ms}$ blocks $(Z=-3.0239, p=0.0160$ for comparison with $180 \mathrm{~ms}, Z=-2.9493, p=0.0220$ with $540 \mathrm{~ms}$ ) during the washout trials. However, this improvement in solo trials could be connected to the sequence of blocks and, therefore, to the fact that with growing number of trials subjects have more training: it is possible that in washout (solo) trials after the last two blocks (180 and $540 \mathrm{~ms}$ delay) subjects are simply more skilled.

Cross-correlation delay (see Figure 3C), which describes the resulting time delay between the target and subject positions, was also significantly influenced by the RP's delay $\left(\chi^{2}(5)=35.89, p<0.0001\right)$. The cross-correlation delay was reduced during the interaction with the RP comparing to solo trials $(Z=3.098, p=0.021)$. When completing the task with small delays of $20-60 \mathrm{~ms}$, the cross-correlation delay was significantly smaller than in the solo condition $(Z=3.1733, p=0.008$ between solo and $20 \mathrm{~ms}$ and $Z=3.1359, p=0.009$ between solo and $60 \mathrm{~ms}$ blocks). The values were not statistically different between the 20$60 \mathrm{~ms}$ delay blocks $(p>0.9$ for all pairwise comparisons between them).
The results suggest that haptic human-robot interaction with delays within $60 \mathrm{~ms}$ does not impede the timing performance of participants compared to their solo performance. Further growth of the RP delay resulted in increase of the cross-correlation delay comparing to smaller delay values $(Z=-3.1733, p=0.008$ between 20 and $540 \mathrm{~ms}$, $Z=-3.6028, p=0.004$ between 60 and $180 \mathrm{~ms}$ and $Z=-3.5096, p=0.005$ between 60 and $540 \mathrm{~ms}$ blocks).

\section{Muscle co-contraction}

Do the subjects change their effort levels throughout the experiment? To analyse effort across the trials, $E M G$ co-contraction was considered. Figure 3D depicts the subject's co-contraction with respect to the different blocks. A Friedman test revealed a significant effect of delay on the co-contraction values $\left(\chi^{2}(5)=40.829, p<0.0001\right)$. Co-contraction initially diminishes from its value during solo performance to those working with a robot $(Z=$ $2.9493, p=0.02$ for comparison between solo and $60 \mathrm{~ms}$ delay groups, other comparisons were not significant $p>$ $0.08)$. The co-contraction remains unchanged for delay conditions between $0-60 \mathrm{~ms}$ (all $p>0.1$ ) and then increases with the increase in delay for both the $180 \mathrm{~ms}$ and $540 \mathrm{~ms}$ delay blocks $(Z=-2.9866, p=0.017$ between 20 and $180 \mathrm{~ms}, Z=-3.4719, p=0.002$ between 20 and $540 \mathrm{~ms}$, $Z=-3.8826, p<0.0001$ between $60 \mathrm{~ms}$ and $180 \mathrm{~ms}$, 

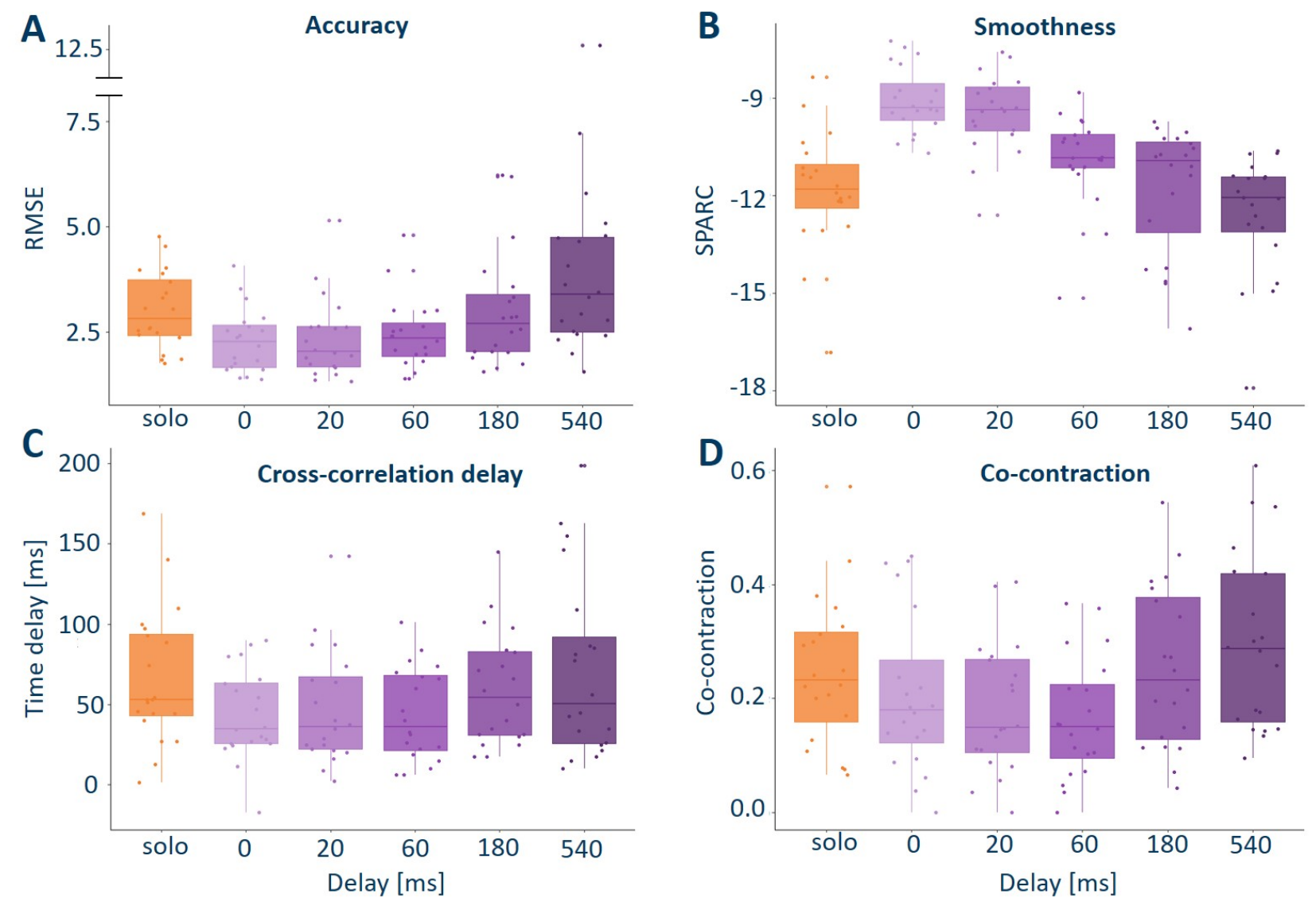

Fig. 3. Performance and EMG activation results. Individual dots display an individual subjects average performance/co-contraction within a block. The box plot then shows the median for each condition, in addition to the upper and lower quartiles of the data. A: Subject accuracy. B: Subject smoothness. C: Cross-correlation delay. D: Subject co-contraction.

$Z=-3.9199, p<0.0001$ between 60 and $540 \mathrm{~ms}$, as well as $Z=-2.9866, p=0.017$ between 180 and $540 \mathrm{~ms}$ delay blocks). Interestingly, we found the lowest overall cocontraction for the $60 \mathrm{~ms}$ delay condition. One possible explanation is that this is due to the human reaction delay which was close to $60 \mathrm{~ms}$, as observed from the solo trials (median $M=60$, median absolute deviation $M A D=44$ ), thus minimising torque exchange during the interaction.

\section{DISCUSSION}

Could the subjects identify delayed force exchange during haptic interaction? Our results suggest that subjects can: while they recognise a change already with a $20 \mathrm{~ms}$ delay, at $60 \mathrm{~ms}$ and beyond they clearly perceive the effect as delay (median $M=3.5$ for $60 \mathrm{~ms}, M=4$ for both $180 \mathrm{~ms}$ and $540 \mathrm{~ms}$ delay groups). This significant difference shows that the subjects are aware that something has changed and recognise delay as a likely cause even with small delay times.

The response to recognising haptic resistance appears to follow a similar trend to that of the recognition of delay. It is unclear if this result reflects an association between delay and resistance, however, it does match observed results which suggest that subjects can perceive delay as an alteration of impedance properties, where haptic delay can lead to increased resistance and visual delay reduced resistance [18], [19]. This may be caused by the subjects misinterpreting the force delay (2) to instead be using the correct position information with altered impedance properties. In contrast to previous results, subjects did not clearly perceive a change in their effective mass [18]. It is unclear if this difference follows from perception of the human-like robot partner or is impacted by our particular choice of input frequencies.

Despite showing the ability to recognise force delay, subjects were not able to account for its impact for values larger than or equal to $180 \mathrm{~ms}$. For the delay values prior to $180 \mathrm{~ms}$, however, we observe that the subjects appear to possess equivalent performance. From these results, it is not clear if the subjects are actively altering their movement strategy. However, it can be observed from the smoothness that the overall subject smoothness at $60 \mathrm{~ms}$, which represents the point at which subjects clearly recognise delay is closer in smoothness to that of the solo interaction. In [5] it was observed that working with the human-like robot controller typically resulted in smoother motion compared to solo and human-human interactions. Therefore the change in smoothness suggests that the human subject may have changed their strategy to more actively override the robot for fine control. 
The results further suggest that co-contraction increases from the $180 \mathrm{~ms}$ delay, at which the subject performance begins to decline. An increase in co-contraction reflects a stiffening of the arm that may help dealing with the perturbation due to delay [20]. This increase in co-contraction also matches the questionnaire data for noise which is identified by subjects at these delay levels. As a result, it is unclear if the perception of noise is the cause of the co-contraction or if instead the co-contraction reflects a strategy change to compensate for increased delay which is then associated with increased noise.

To investigate this effect and to understand the potential changes in EMG, we computed the cross-correlation delay between the target and subjects' position during the solo trials. The participants' delay with respect to target was very close to $60 \mathrm{~ms}$ (median $60 \mathrm{~ms}$, median absolute deviation $44 \mathrm{~ms}$ ). Therefore, at $60 \mathrm{~ms}$ the robot is providing force synchronised to the human motion, which may explain the minimum co-contraction observed at this delay value and the smoothness being more similar to the without robot assistance (solo condition).

The current study makes use of a human-like agent and a constant force delay presented in a fixed order of increasing blocks. While subjects are not able to discriminate between this agent and a human partner, the findings of [5] do indicate that there are subtle differences in the perception of motion compared to a human partner. Whether or not some of these factors including perceived smoothness may alter the results is not known. The robotic partner has been used in this study since it allows for a one-way delay to separate the subjects from each other and have a controllable partner. The use of increasing delay may also bias the results inter alia due to a potential learning effect in later blocks. This decision was made to ensure consistency within the sample: each subject has the same sequence of experimental blocks. Both of these features are atypical for how true haptic communication would work and therefore need to be tested further: for instance through testing with randomised blocks and an investigation into the effect of delay during humanhuman interaction. Furthermore, variable delay and delays only of position information could also occur in typical communication and will be considered in future work.

Although we observe performance degradation, it should be noted that the subject's performance with force delay retains benefits of haptic communication relative to solo performance until $540 \mathrm{~ms}$. Furthermore subjects found the feedback from the haptic communication to be assisting in general for these conditions and only suggested that they were not being assisted after $540 \mathrm{~ms}$. While the exact magnitude of the allowable amount of delay for improved performance is likely a function of both the relative subject ability and the trajectory dynamics, in accordance with the observed trajectory tracking results for visual delay [10], the results do suggest some robustness in human haptic communication. The characteristics of this robustness merit further investigation to determine how humans model the effect of delay into the communicated information.

\section{REFERENCES}

[1] K. B. Reed and M. A. Peshkin, "Physical collaboration of humanhuman and human-robot teams," IEEE Transactions on Haptics, vol. 1, no. 2, pp. 108-120, 2008.

[2] G. Ganesh, A. Takagi, R. Osu, T. Yoshioka, M. Kawato, and E. Burdet, "Two is better than one: Physical interactions improve motor performance in humans," Scientific Reports, vol. 4, p. 3824, 2014.

[3] D. A. Abbink, T. Carlson, M. Mulder, J. C. de Winter, F. Aminravan, T. L. Gibo, and E. R. Boer, "A topology of shared control systems-finding common ground in diversity," IEEE Transactions on Human-Machine Systems, vol. 48, no. 5, pp. 509-525, 2018.

[4] A. Takagi, G. Ganesh, T. Yoshioka, M. Kawato, and E. Burdet, "Physically interacting individuals estimate the partner's goal to enhance their movements," Nature Human Behaviour, vol. 1, no. 3, p. 0054, 2017.

[5] E. Ivanova, G. Carboni, J. Eden, J. Krueger, and E. Burdet, "For motion assistance humans prefer to rely on a robot rather than on an unpredictable human," IEEE Open Journal of Engineering in Medicine and Biology, 2020.

[6] G. C. Goodwin, S. F. Graebe, M. E. Salgado et al., Control system design. Upper Saddle River, NJ: Prentice Hall,, 2001.

[7] J. Guo, C. Liu, and P. Poignet, "Stable and enhanced position-force tracking for bilateral teleoperation with time delay," in Proc. European Control Conference, 2015, pp. 1980-1985.

[8] A. C. Smith and K. Hashtrudi-Zaad, "Smith predictor type control architectures for time delayed teleoperation," The International Journal of Robotics Research, vol. 25, no. 8, pp. 797-818, 2006.

[9] S. Friston, P. Karlström, and A. Steed, "The effects of low latency on pointing and steering tasks," IEEE Transactions on Visualization and Computer Graphics, vol. 22, no. 5, pp. 1605-1615, 2015.

[10] A. Pavlovych and W. Stuerzlinger, "Target following performance in the presence of latency, jitter, and signal dropouts." in Graphics Interface, vol. 2011, 2011, pp. 33-40.

[11] I. S. MacKenzie and C. Ware, "Lag as a determinant of human performance in interactive systems," in Proc. INTERACT and CHI Conference on Human Factors in Computing Systems, 1993, pp. 488493.

[12] C. Jay, M. Glencross, and R. Hubbold, "Modeling the effects of delayed haptic and visual feedback in a collaborative virtual environment," ACM Transactions on Computer-Human Interaction (TOCHI), vol. 14 , no. 2 , pp. 8-es, 2007.

[13] M. Ferraz, E. Ferreira, E. den Exter, F. van der Hulst, H. Rovina, W. Carey, J. Grenouilleau, and T. Krueger, "Multisensory real-time space telerobotics," in Intelligent Computing-Proceedings of the Computing Conference, 2019, pp. 275-298.

[14] R. C. Oldfield, "The assessment and analysis of handedness: the edinburgh inventory," Neuropsychologia, vol. 9, no. 1, pp. 97-113, 1971.

[15] A. Melendez-Calderon, L. Bagutti, B. Pedrono, and E. Burdet, "Hi5: A versatile dual-wrist device to study human-human interaction and bimanual control," in Proc. IEEE/RSJ Conf. on Intelligent Robots and Systems (IROS), 2011, pp. 2578-2583.

[16] A. Takagi, M. Hirashima, D. Nozaki, and E. Burdet, "Individuals physically interacting in a group rapidly coordinate their movement by estimating the collective goal," eLife, vol. 8, p. e41328, 2019.

[17] S. Balasubramanian, A. Melendez-Calderon, A. Roby-Brami, and E. Burdet, "On the analysis of movement smoothness," Journal of Neuroengineering and Rehabilitation, vol. 12, no. 1, pp. 1-11, 2015.

[18] W. Fu, M. M. van Paassen, D. A. Abbink, and M. Mulder, "Framework for human haptic perception with delayed force feedback," IEEE Transactions on Human-Machine Systems, vol. 49, no. 2, pp. 171$182,2018$.

[19] B. Knorlein, M. Di Luca, and M. Harders, "Influence of visual and haptic delays on stiffness perception in augmented reality," in IEEE International Symposium on Mixed and Augmented Reality, 2009, pp. $49-52$.

[20] D. W. Franklin, R. Osu, E. Burdet, M. Kawato, and T. E. Milner, "Adaptation to stable and unstable dynamics achieved by combined impedance control and inverse dynamics model," Journal of Neurophysiology, vol. 90, no. 5, pp. 3270-3282, 2003. 